ESAIM: PROCEEDINGS AND SURVEYS, February 2015, Vol. 49, p. 78-90

Samira EL YACOUBI, Larbi AFIFI, El Hassan ZERRIK, Abdessamad TRIDANE, Editors

\title{
SIMPLE FISHERY AND MARINE RESERVE MODELS TO STUDY THE SLOSS PROBLEM
}

\author{
A. Moussaoui ${ }^{1}$ And P. Auger ${ }^{2}$
}

\begin{abstract}
Habitat fragmentation is generally believed to be detrimental to the persistence of natural populations. In nature management one therefore tends to prefer many small nature reserves over one single large having equal total area. The paper presents two tractable analytical models to examine whether this preference is warranted in a metapopulation framework with reserves (patches) by formulating the dependence of the density as well as the global catch on the number of reserves. Studying these models, we seek to compare the two different strategies: whether a single large reserve will (1) conserve more species and (2) maintains high yield in fisheries than several small or vice versa? Our results indicate that it is favourable to make several small reserves instead one single large reserve. Indeed, at equilibrium, the density as well as the global catch are bigger in the Several Small (SS) strategy than in the Single Large (SL) one. In addition, in the case of SS strategy, an intermediate reserve number exists which is optimal for catch at equilibrium. The results in this paper may provide important implications for nature conservation.
\end{abstract}

Résumé. La fragmentation de l'habitat est généralement considéré comme préjudiciable à la persistance des populations naturelles. Dans la gestion des milieux naturels, il y a donc tendance à préférer plusieurs petites réserves à une seule ayant la même superficie. Ce papier présente deux modèles mathématiques étudiant ce problème dans le cadre d'une métapopulation avec des zones protégées représentés par des sites discrets. Nous étudions notamment les captures globales en fonction du nombre de réserves. Nous cherchons par cette étude à comparer deux stratégies de préservation : Une seule grande réserve ou plusieurs petites. Nos résultats indiquent qu'il est préférable de mettre plusieurs petites réserves plutôt qu'une seule réserve. En effet, à l'équilibre, la densité ainsi que la capture globale sont plus grandes dans le cas de la stratégie avec plusieurs petites réserves (SS) que dans le cas d'une seule grande (SL). En outre, dans le cas de la stratégie SS, nous démontrons qu'il existe un nombre optimal de reserves qui maximise la capture totale à l'équilibre. Les résultats présentés dans cette contribution sont susceptibles d'avoir des implications importantes dans le domaine de la conservation des espèces.

\section{INTRODUCTION}

Marine reserves, or no-take zones, have been recently promoted as a means of managing marine populations for two different goals: preserving biodiversity [1], and managing fisheries [19] to produce the highest yields. Controversy on how to plan marine reserves lead scientists to start the debate on whether single large or several small reserves would be better to conserve biodiversity [16,17], this is known in the ecological literature as the Single Large Or Several Small (SLOSS) problem. The point of view that a single large reserve is

${ }^{1}$ Department of Mathematics, Faculty of Sciences, University of Tlemcen, 13000, Algeria.

2 UMI IRD 209 UMMISCO, Centre IRD de llle de France, 32 Avenue Henri Varagnat, 93143 Bondy Cedex, France.

UPMC, Sorbonne University Pierre et Marie Curie-Paris 6, France.

(C) EDP Sciences, SMAI 2015 
the best and most intuitive strategy for long-term population persistence is mainly based on species area relationships. However, the concept of "keeping all eggs in the same basket" makes the single large argument weaker, considering the risks involved in allocating all investments in one single area [20]. By the other side, several small areas make populations easy targets for demographic and environmental stochasticity, and effects of genetic degeneration (i.e. inbreeding and bottleneck effects). However, it is also expected that by maintaining several areas, higher habitat variation and, as consequence, higher species richness are achieved at landscape scale.

Since the beginning of the SLOSS debate in the middle of 1970s, researchers are testing hypotheses considering different landscape scenarios and distinct taxonomic groups in an attempt to provide a final answer to such dichotomy.

Although a simple model cannot provide a definitive answer to this question, it can be used to explore several possibilities. Our goal in this paper is to look in a quantitative this question within the context of two simple deterministic models. First of all, we study the dynamics of a model in the case of no reserve. We then generalize to the case of marine reserve in section 3. The model includes two time scales, a fast one associated to quick movements of fish between sites in comparison to a slow one corresponding to the growth of the fish population and the change of the fleet size. We take advantage of these two time scales to build a reduced model, by applying aggregation methods of variables [6,9]. The reduced model, called aggregated model, describes the dynamics of the total fish stock and the fishing effort. Studying this aggregated model, we investigate the practical effects of the protection zone on the conservation of population resources and catch at equilibrium. In section 4, we propose a dynamic one-dimensional model of Marine Protected Areas (MPAs) along a coastline to investigate the practical effects of several small reserve on the conservation of population resources and catch at equilibrium. In section 5, we then compare results obtained, particularly using numerical simulations. Our results indicate that having multiple small MPAs may be preferable to having a single large one of the same area.

\section{Classical Fishery MODEL}

In this section we summarize some crucial properties of classical fishery models without marine reserves. This will serve as a convenient benchmark in evaluating the impact of marine reserves in later sections. Consider a fishing area of constant size $K$ inhabited by a stock of fish. Let the stock of fish at each point of time be fully described by its biomass, $n(t)$ where $t$ refers to time and $E(t)$ be the fishing effort, at time $t$. We assume that the population evolves according to the logistic law of growth. The following system describes the time evolution of the fishery [26]:

$$
\left\{\begin{array}{l}
\frac{d n}{d t}=r n\left(1-\frac{n}{K}\right)-q n E \\
\frac{d E}{d t}=(-c+p q n) E
\end{array}\right.
$$

where $r$ is the growth rate of the resource. It is also assumed that the catch is proportional to the fish density and to the fishing effort. $q>0$ is a catchability parameter, $c$ represents the cost per unit of fishing effort, and $p$ is the price per unit of fish. This system is well know because it is equivalent to a Lotka-Volterra predator-prey model with logistic growth for prey. It has three equilibria, $(0,0),(K, 0)$, which is a "fishery-free" equilibrium $(F F E)$, and a unique and possibly positive fishery equilibrium $(\mathrm{FE})\left(n^{*}, E^{*}\right)$ with

$$
\left\{\begin{array}{l}
n^{*}=\frac{c}{p q} \\
E^{*}=\frac{r}{q}\left(1-\frac{c}{p q K}\right)
\end{array}\right.
$$


This equilibrium is globally asymptotically stable when $p q K>c$, in this case, the fishery is viable and the catch per unit of time at equilibrium is

$$
Y^{*}=q n^{*} E^{*}=\frac{r c}{p q}\left(1-\frac{c}{p q K}\right)
$$

Keeping all parameters constant except the catchability parameter $q$, one sees from equation (3) that $Y^{*}$ is a function of $q$. $Y^{*}$ is positive when $q \in\left[\frac{c}{p K}, \infty\left[\right.\right.$ and reaches a maximum value for $q=\frac{2 c}{p K}$. for this value of $q$, the optimal catch at equilibrium is $\frac{r K}{4}$.

\section{Single LARGe MPA}

We now turn our attention to marine reserves. We begin by considering the impact on the previous classical fishery model. We assume that fishing is prohibited in a certain part of the coast. Let $s$ be the fraction of the protected zone of the total coast. Clearly, being a fraction $s \in[0,1] . \quad s=0$ there is no marine reserve and $s=1$ indicates that the whole fishing area is a reserve. Then, we assume that this area splits in two sub-zones, with capacity respectively equal to $k_{1}=s K$ and $k_{2}=(1-s) K$, we denote the stock of the corresponding sub-populations by $n_{1}$ and $n_{2}$. These two populations follow two independent evolution laws and this is the reason why we use the "patch concept". From now on, we decide that the first patch with capacity $k_{1}$ is a protected zone where no catch is allowed, whereas in the second patch fishing is allowed (Figure 1). We now

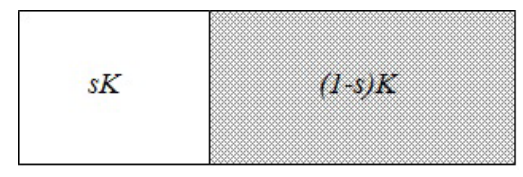

FiguRE 1. Schematic representation of a network of marine reserve with reserve in white and fished area in gray.

assume that some exchange of fishes exists between these two patches and that this can occur at a fast time scale $\tau$, whereas fish growth and the dynamics of the fishery occur at a slow time scale $t=\epsilon \tau$ with $\epsilon<<1$ being a small dimensionless parameter. It makes sense to assume that fish movement rates are inversely proportional to that carrying capacity of the site they leave $[4,10,22,23]$ :

$$
m_{i}=\frac{\sigma}{k_{i}}
$$

where $m_{1}$ and $m_{2}$ are the migration rates from patch 1 to patch 2 and from patch 2 to patch 1 respectively, and $\sigma$ is a positive constant that represents the migration rate when the carrying capacity of the departure site is set to 1. Thus, according to Eq. 4, fish are more likely to remain on sites offering large carrying capacities, i.e., on sites where the resource is abundant. Using the same parameters $r, q, c$, and $p$ as in the classical fishery model. The complete system, at the fast time scale $\tau$ with respect to $t$, reads as follows

$$
\left\{\begin{array}{l}
\frac{d n_{1}}{d \tau}=\left(m_{2} n_{2}-m_{1} n_{1}\right)+\varepsilon r n_{1}\left(1-\frac{n_{1}}{s K}\right) \\
\frac{d n_{2}}{d \tau}=\left(m_{1} n_{1}-m_{2} n_{2}\right)+\varepsilon\left(r n_{2}\left(1-\frac{n_{2}}{(1-s) K}\right)-q n_{2} E\right) \\
\frac{d E}{d \tau}=\varepsilon\left(-c+p q n_{2}\right) E
\end{array}\right.
$$




\subsection{The agregated model}

From the complete system (5), we apply aggregation methods to obtain a reduced system: a two dimensional system of ordinary differential equations governing the total fish stock and the total fishing effort at the slow time scale. The aggregation of the complete model consists in supposing that the fast dynamics has attained a stable equilibrium and in substituting this fast equilibrium into the equations of the complete model. The first step to achieve aggregation is to neglect the small terms of the order of $\varepsilon$ in Eq.(5) and to look for the existence of stable equilibria for its fast part (see $[3,6,9,24]$ and the references therein for more details about this method).

Let $n=n_{1}+n_{2}$ be the total fish population, the previous system can be written as follows:

$$
\left\{\begin{array}{l}
\frac{d n_{2}}{d \tau}=\left(m_{1} n-\left(m_{1}+m_{2}\right) n_{2}\right)+\varepsilon\left(r n_{2}\left(1-\frac{n_{2}}{(1-s) K}\right)-q n_{2} E\right) \\
\frac{d n}{d \tau}=\varepsilon\left(r n\left(1-\frac{n-n_{2}}{s K}\right)+r n_{2}\left(\frac{(1-s) n-n_{2}}{s(1-s) K}\right)-q n_{2} E\right) \\
\frac{d E}{d \tau}=\varepsilon\left(-c+p q n_{2}\right) E
\end{array}\right.
$$

The aggregated model is obtained by considering the equilibrium of the fast part:

$$
n_{2}^{*}=\frac{m_{1}}{m_{1}+m_{2}} n=(1-s) n
$$

and by replacing $n_{2}$ by this expression in the slow part, which gives the following aggregated model:

$$
\left\{\begin{array}{l}
\frac{d n}{d t}=r n\left(1-\frac{n}{K}\right)-Q n E \\
\frac{d E}{d t}=(-c+p Q n) E
\end{array}\right.
$$

where $Q$ (the global catchability parameter) is given by

$$
Q=q(1-s)
$$

The system (7) is equivalent to the no reserve model (Eq. 1) except that the size $s$ appears in the equations via the parameter $Q$. A direct application of the results obtained for the no-reserve model therefore leads to the following results: Aggregated model $(7)$ has a unique positive fishery equilibrium $\left(n^{*}, E^{*}\right)$ such that

$$
\left\{\begin{array}{l}
n^{*}=\frac{c}{p Q}=\frac{c}{p q(1-s)} \\
E^{*}=\frac{r}{Q}\left(1-\frac{c}{p Q}\right)=\frac{r}{q(1-s)}\left(1-\frac{c}{p q(1-s) K}\right)
\end{array}\right.
$$

Without the reserve $(s=0)$, a nonnegative $E^{*}$ implies the restriction $\frac{c}{p q K}<1$ When the reserve is present, a further restriction is

$$
s<1-\frac{c}{p q K}=s_{\max } .
$$

For the stability analysis, the following two cases occur:

- (i) If $s>1-\frac{c}{p q K}$, the $\mathrm{FE}\left(n^{*}, E^{*}\right)$ is not positive, and the $\mathrm{FFE}(K, 0)$ is globally asymptotically stable.

- (ii) If $s<1-\frac{c}{p q K}$, the FE is positive and globally asymptotically stable, and the FFE is unstable (saddle point).

To make some headway it may be useful to resort again to a numerical representation of the situation. Indeed, as will become evident below in Figure 2, marine reserves enhance the biomass growth function, this is the main justification for introducing marine reserves. Marine reserves may influence biomass growth in various 
ways. It is for instance possible that marine reserves, by providing some of the fish with a sanctuary from disturbing fishing activity may actually enhance biomass growth.

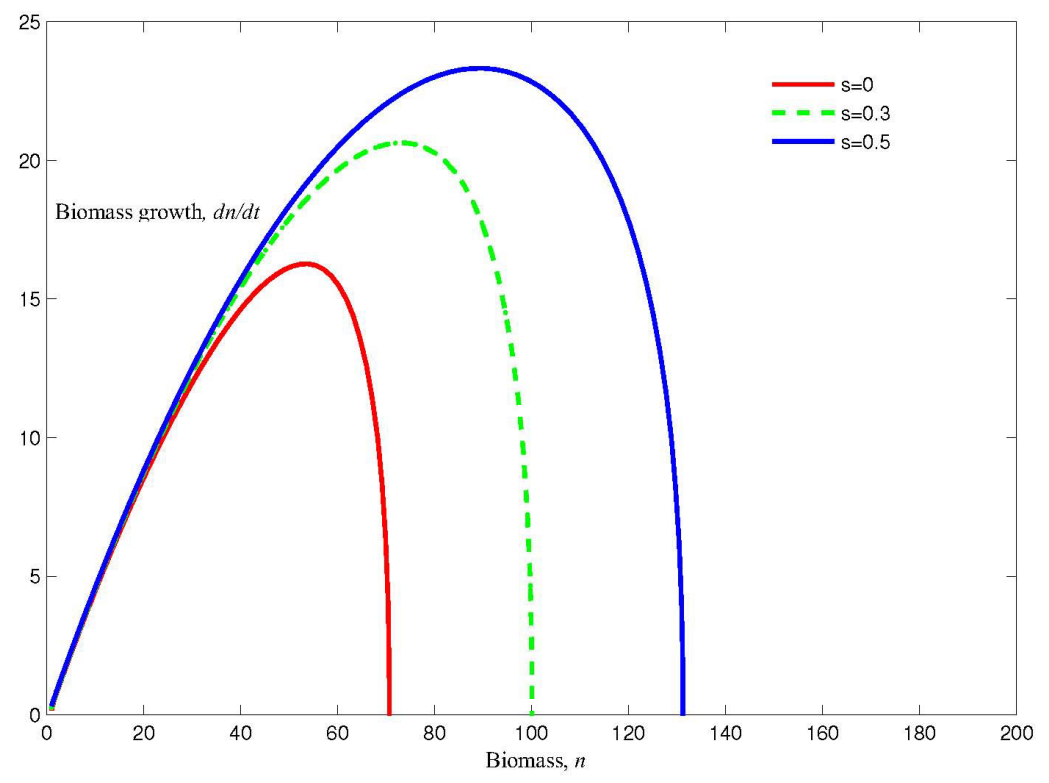

FIGURE 2. The effect of marine reserves on biomass growth: $r=0.5, k=200, q=0.17, p=$ $0.15, c=0.5$.

\subsection{The Optimal Size of a Marine Reserve}

Given the above specifications, it is possible to derive expressions for the optimal size of marine reserves. The catch per unit of time at equilibrium is given by

$$
Y^{*}=r n^{*}\left(1-\frac{n^{*}}{K}\right)=\frac{r c}{p q(1-s)}\left(1-\frac{c}{p q(1-s) K}\right)
$$

Equation (11) gives the harvesting taking place in the non-reserve area as a function of the size of the reserve area. To find the optimal size, keeping all parameters constant except $s$, equation (11) is expanded into a negative quadratic function of $s$. The optimal value for the parameter $s$ which maximizes the stationary catch $Y^{*}$ is given by

$$
s_{S L}^{*}=1-\frac{2 c}{p q K}
$$

The optimal reserve size $s^{*}$ as calculated in equation (12) is clearly less than $s_{\max }$. In other words, $s^{*}$ is economically feasible (Figure 4). We can also note that for $s=s^{*}$ we have $n^{*}=\frac{K}{2}$ and $Y^{*}=\frac{r K}{4}$.

\section{Several SMAll MPAs}

In order to investigate the effects of space partitioning in the efficacy of MPAs, we replicated the abovedescribed scenarios with several smaller MPAs totalling the surface area of the single MPA. Our model assumes 
a linear array of $2 L$ areas such as might occur along a coastline (Figure 3 ). We assume that between two consecutive fishing patches, there is always a single non fishing patch, which is a fish refuge. Each patch is relatively isolated, but a fish population migrates between any pair of adjacent patches and boat displacements between any pair of adjacent fishing patches. We assume that fish movements and boat displacements occur at a fast time scale $\tau$, whereas fish growth and the dynamics of the fishery occur at a slow time scale $t=\varepsilon \tau$, with $\varepsilon<<1$ being a small dimensionless parameter. Each reserve sites $i(i=1, \ldots, L)$ has a carrying capacity $k_{2 i-1}=s_{2 i-1} K$, and the total size of these reserves equal to $s K$ where $s=\sum_{i=1}^{L} s_{2 i-1}<1$. It makes sense to assume that the movement rate for boats from a site to a neighboring site depends on the distance between these sites. Therefore, we assume symmetric movement rates for boats:

$$
\beta_{2 i-1,2 i+1}=\beta_{2 i+1,2 i-1}
$$

for $i \in[1, L-1]$. Fish movement rates are inversely proportional to the carrying capacity of the site they leave:

$$
m_{i+1, i}=\frac{\sigma}{k_{i}} \quad \text { for all } i \in[1,2 L]
$$

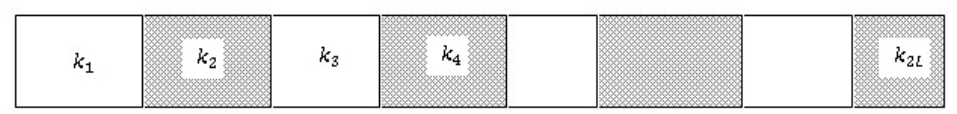

FIGURE 3. Schematic representation of a network of marine reserves with reserves in white and fished areas in gray, graph in a linear set of patches having a total number of patches $2 L$, with $L$ fishing $(F)$ patches and $L$ non fishing $(N F)$ patches.

Let $n_{i}(t)$ be the biomass of fish on patch $i$ and $E_{i}(t)$ be the fishing effort at time $t$, i.e. the number of fishing boats on patch $i$ at time $t$, using the same parameters $r, q, c$ and $p$, the next system that we call the complete system describes the time evolution of the fishery at patch $i$ :

$$
\left\{\begin{array}{r}
\frac{d n_{i}}{d \tau}=m_{i, i-1} n_{i-1}+m_{i, i+1} n_{i+1}-\left(m_{i-1, i}+m_{i+1, i}\right) n_{i}+\varepsilon\left(r n_{i}\left(1-\frac{n_{i}}{k_{i}}\right)-q n_{i} E_{i}\right) \\
\text { if area } i \text { is not in a reserve } \\
\frac{d n_{i}=\left(m_{i, i-1} n_{i-1}+m_{i, i+1} n_{i+1}-\left(m_{i-1, i}+m_{i+1, i}\right) n_{i}\right)+\varepsilon r n_{i}\left(1-\frac{n_{i}}{k_{i}}\right)}{\text { if area } i \text { is in a reserve }}
\end{array}\right.
$$

for $i \in[2,2 L-1]$.

For the first and last patches we have:

$$
\left\{\begin{array}{c}
\frac{d n_{1}}{d \tau}=\left(m_{12} n_{2}-m_{21} n_{1}\right)+\varepsilon\left(r n_{1}\left(1-\frac{n_{1}}{k_{1}}\right)-q n_{1} E_{1}\right) \\
\frac{d n_{2 L}}{d \tau}=\left(m_{2 L, 2 L-1} n_{2 L-1}-m_{2 L-1,2 L} n_{2 L}\right)+\varepsilon r n_{2 L}\left(1-\frac{n_{2 L}}{k_{2 L}}\right)
\end{array}\right.
$$

We assume that fish have the same growth rates on the fishing site and on the reserve site, Boats are allocated to areas based on the abundance of fish in the areas outside the reserve, no vessels fish inside the reserve. The 
following system describes the time evolution of the fishing efforts along the chain at the fast time scale:

$$
\left\{\begin{array}{cc}
\frac{d E_{i}}{d \tau}=\left(\beta_{i, i-2} E_{i-2}+\beta_{i, i+2} E_{i+2}-\left(\beta_{i-2, i}+\beta_{i+2, i}\right) E_{i}\right)+\varepsilon\left(-c+p q n_{i}\right) E_{i} \\
\text { if area } i \text { is not in a reserve } \\
\frac{d E_{i}}{d \tau}=0 & \text { if area } i \text { is in a reserve }
\end{array}\right.
$$

for $i \in[3,2 L-3]$.

For the first and last fishing patches we have:

$$
\left\{\begin{array}{l}
\frac{d E_{1}}{d \tau}=\left(\beta_{13} E_{3}-\beta_{31} E_{1}\right)+\varepsilon\left(-c+p q n_{1}\right) E_{1} \\
\frac{d E_{2 L-1}}{d \tau}=\left(\beta_{2 L-1,2 L-3} E_{2 L-3},-\beta_{2 L-3,2 L-1} E_{2 L-1}\right)+\varepsilon\left(-c+p q n_{2 L-1}\right) E_{2 L-1}
\end{array}\right.
$$

\subsection{The aggregated model}

Now, we shall take advantage of aggregation of variables methods to build a reduced model $[4,9]$. The first step is to set $\varepsilon=0$ in the previous complete model and to look for existence and stability of a fast equilibrium which is as follows (see appendix A):

$$
\left\{\begin{array}{l}
n_{i}^{*}=v_{i}^{*} n=\frac{k_{i}}{K} n \\
E_{i}^{*}=\mu_{i}^{*} E=\frac{1}{L} E \text { if area } i \text { is not in a reserve } \\
E_{i}^{*}=0 \text { if area } i \text { is in a reserve }
\end{array}\right.
$$

where $n=\sum_{i=1}^{2 L} n_{i}$ is the total biomass of fish and $E=\sum_{i=1}^{L} E_{2 i-1}$ is the total fishing effort.

Substituting the fast equilibrium into the complete model leads after some straightforward calculation to the fishery two-dimensional aggregated model which reads as follows (see appendix B):

$$
\left\{\begin{array}{l}
\frac{d n}{d t}=r n\left(1-\frac{n}{K}\right)-Q n E \\
\frac{d E}{d t}=(-c+p Q n) E
\end{array}\right.
$$

where $Q$ (global catchability parameter) is given by

$$
Q=\frac{q(1-s)}{L}
$$

The system (20) is equivalent to the no reserve model (Eq. 1) except that the number $L$ and the size $s$ appears in the equations via the parameter $Q$. A direct application of the results obtained for the single large model therefore leads to the following results: The multisite aggregated model (Eq. 20) has a unique positive fishery equilibrium $\left(n^{*}, E^{*}\right)$ such that

$$
\left\{\begin{array}{l}
n^{*}=\frac{c}{p Q}=\frac{c L}{p q(1-s)} \\
E^{*}=\frac{r}{Q}\left(1-\frac{c}{p Q}\right)=\frac{r L}{q(1-s)}\left(1-\frac{c L}{p q(1-s) k}\right)
\end{array}\right.
$$

The fishery is viable provided that this equilibrium belongs to the positive quadrant, i.e.:

$$
s<1-\frac{c L}{p q K}=s_{\max }
$$


The catch $Y^{*}$ of the fishery at equilibrium is given by

$$
Y^{*}=Q n^{*} E^{*}=\frac{r c L}{p q(1-s)}\left(1-\frac{c L}{p q(1-s) K}\right)
$$

We now turn to problem of the optimal size of reserves that maximizes the catch at equilibrium under a fixed number of MPAs.

From (24), we see that $Y^{*}$ has a maximum for the following size of reserves:

$$
s_{S S}^{*}=1-\frac{2 c L}{p q K}
$$

We can also note that for $s=s_{S S}^{*}$, we have $n^{*}=\frac{K}{2}$ and $Y^{*}=\frac{r K}{4}$. (Figure 4).

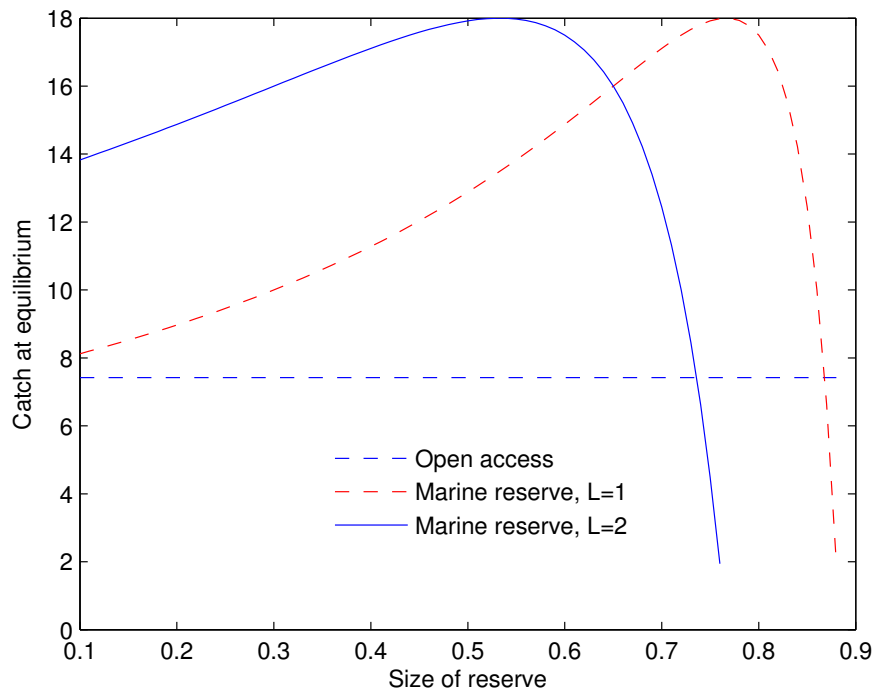

FiguRE 4. Effects on catch at equilibrium of varying reserve size.

From Figure 4, it becomes evident that there exists a positively size optimal marine reserve that will maximize the value of the fishery. This can be seen by noting that the value of the fishery increases as $s$ begins to increase. However, it is important to note that the increase in value does not persist. As the size of the reserve becomes too large, it restricts the available fishery population for harvest and therefore reduces the value of the fishery. As illustrated in Figure 5, the introduction of marine reserves uniformly increases the biomass of fish at equilibrium. The reason is that marine reserves enhance biological growth of fish.

Figure 6 represents the behavior of the fish population over time, with and without reserve, Figure 6 show that the effect of the reserve on 'stability' depends on how stability is measured. In all cases a reserve improves convergence, however, reserves tend to make oscillations more rapid during convergence, we observe also that making several MPAs stabilize more rapidly the system.

\subsection{The Optimal Number of Marine Reserves}

We no examine the question of many reserve sites that should be established to maximize the catch at equilibrium given a total reserve size as constant. Since the catch is a quadratic function of number of reserve 


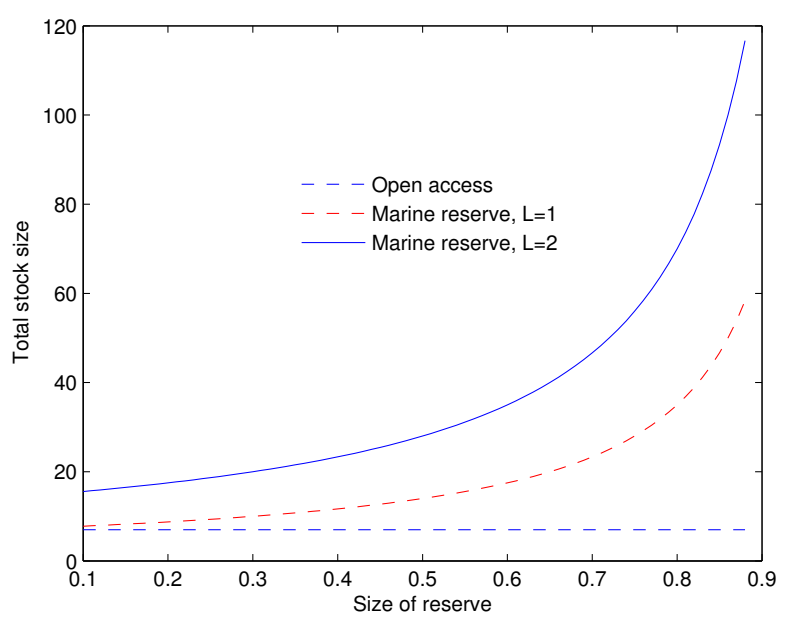

FIGURE 5. Effect on stock of varying reserve size.
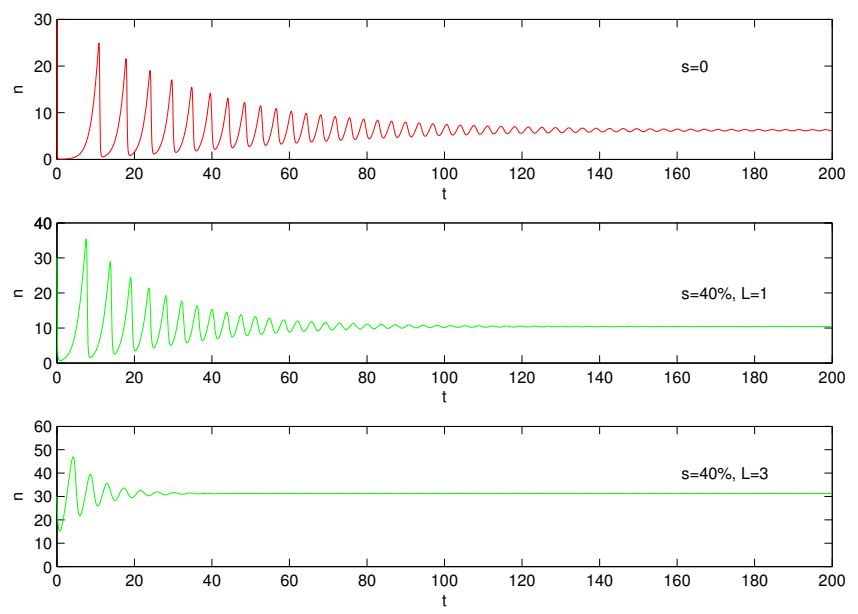

FiguRE 6. Dynamic effect of reserve on stock density.

$L$ (Eq. (24). The optimal value for the parameter $L$ which maximizes the stationary catch $Y^{*}$ is given by

$$
L_{o p t}=\frac{p q(1-s) K}{2 c}
$$

We can also note that for $L=L_{\text {opt }}$ we have $n^{*}=\frac{K}{2}$ and $Y^{*}=\frac{r K}{4}$. (Figure 7). From this graphical result, it becomes evident that there exists an optimal number of marine reserves that will maximize the value of the fishery. 


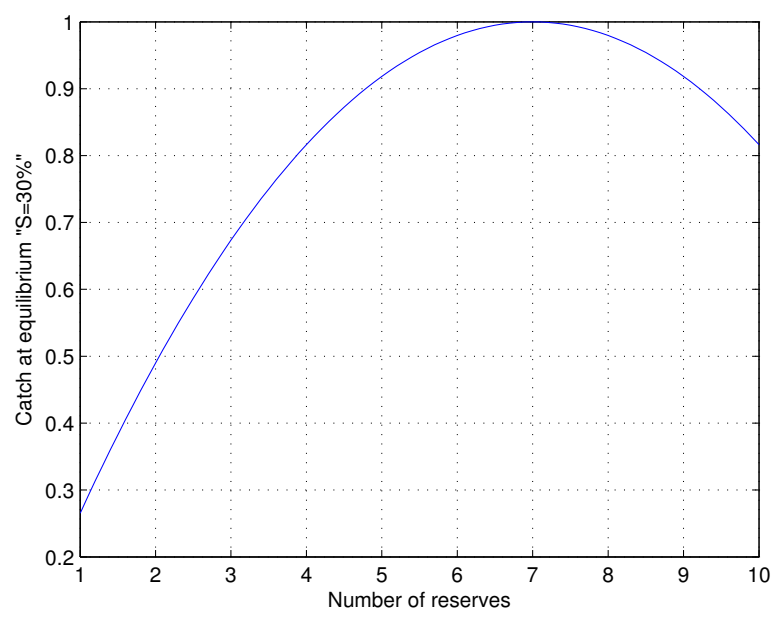

FIGURE 7. Effects of the number of reserves on the catch at equilibrium, $\mathrm{s}=0.3$

\section{Comparaison Between the two scenarios And COnClusion}

One of the most frequently debated theories in conservation biology has been whether the best strategy for species survival is to have a single large or several small remnant refuge patches as nature reserves (SLOSS). The debate has generated considerable controversy [28], and it has been concerned mainly with the question of what configuration could maximize biodiversity [14]. These simple models illustrates that the consequences of MPA implementation on both stock abundance and catch will depend how to allocate the MPA. In the interpretation of the results, the reader should be aware that the analysis focuses on a simplified representation of reality, as does the original SLOSS problem. As Soule and Simberloff [27] point out, the answer to the problem depends strongly on the situation considered. Local differences in environmental quality, as well as geographic variables, such as the distance between reserve sites and the characteristics of the species involved, are extremely important for the optimal number of sites. Diamond [14] stated that a Single Large (SL) reserve was preferred over Several Small (SS) reserves (keeping total area equal) for multispecies conservation. Subsequent theoretical (Higgs and Usher [18]) and empirical (Gilpin and Diamond [16]) work, however, suggested that a large number of sites covers more species than a single large one, given that total reserve area is constant. According to our results, it appears that having multiple small MPAs may be preferable than a single large one: Our results show that the biomass of fish increases when the number of reserves increases and the level of catch at equilibrium in the SS scenario is larger than under SL scenario. The simulation results indicate that an intermediate reserve number exists, which is optimal for catch at equilibrium (Figure 7). As a result, one could conclude erroneously several small reserves to be better than a single large.

Since the relationship between catch and cost is nonlinear (Eq. 24), hence a maximum catch may occur, our model predicted that even if the costs of fishing increase, the catch increase also, when the cost becomes very high, the catch begins to decrease, this can be justified as following: when the cost increases, fishing effort 


\begin{tabular}{|l|c|c|c|}
\hline & No marine reserve & Single Large MPA & Several Small MPAs \\
\hline$n^{* *}$ & $\frac{c}{p q}$ & $\frac{c}{p q(1-s)}$ & $\frac{c L}{p q(1-s)}$ \\
\hline$E^{*}$ & $\frac{r}{p q}\left(1-\frac{c}{p q k}\right)$ & $\frac{r c}{p q(1-s)}\left(1-\frac{c}{p q(1-s) k}\right)$ & $\frac{r c L}{p q(1-s)}\left(1-\frac{c L}{p q(1-s) k}\right)$ \\
\hline$S_{\max }$ & 1 & $1-\frac{c}{p q K}$ & $1-\frac{c L}{p q K}$ \\
\hline$S_{o p t}^{*}$ & 0 & $1-\frac{2 c}{p q K}$ & $1-\frac{2 c L}{p q K}$ \\
\hline$Y_{\max }^{*}$ & $\frac{r K}{4}$ & $\frac{r K}{4}$ & $\frac{r K}{4}$ \\
\hline
\end{tabular}

TABLE 1. Comparison of SL strategy and SS strategy.

(Eq. 22) decreases, in the same time, the biomass of fish increases (Eq. 22); therefore, boats will be able to catch more fish, when the cost becomes very high, rentability of fishery decreases as well as fishing effort, consequently catches also starts to decrease. In order to avoid this situation and maximize catch at equilibrium, our model suggests to reduce the number of reserves to maximize the capture at equilibrium (Eq. 26). The graph illustrating this situation is similar to the one given in Figure 7. Hence, we omitted to show it.

Similarly, the relationship between catch and price is nonlinear (Eq. 24), we observe that when the price increases, the catch increases to reach some level and then it starts to decrease. This can be justified as follows: when the price of fish increases; the fishery becomes more rentable and thus fishing effort increases. But at the same time, the resource decreases (Eq. 22) and when the stock becomes too low, the catch starts in turn to decrease. In order to avoid this situation and to maximize the catch at equilibrium, it is beneficial to increase the number of reserves (Eq. 26), it allows to better protection of the resource.

\section{Appendix}

\section{(A) Calculation of the fast equilibria}

We notice that $n(t)$ and $E(t)$, the global variables are constants of motion of the fast process; migration. Fast equilibria are the solutions of the following system:

$$
\left\{\begin{array}{r}
m_{i, i-1} n_{i-1}+m_{i, i+1} n_{i+1}-\left(m_{i-1, i}+m_{i+1, i}\right) n_{i}=0 \\
\text { for } i \in[2,2 L-1], \\
\beta_{i, i-2} E_{i-2}+\beta_{i, i+2} E_{i+2}-\left(\beta_{i-2, i}+\beta_{i+2, i}\right) E_{i}=0 \\
\text { for } i \in[3,2 L-3]
\end{array}\right.
$$

For $i=1$ and $i=2 L$, the equations of (A.1) simplifies into

$$
\left\{\begin{array}{l}
m_{12} n_{2}-m_{21} n_{1}=0 \\
m_{2 L, 2 L-1} n_{2 L-1}-m_{2 L-1,2 L} n_{2 L}=0
\end{array}\right.
$$

and for $i=3$ and $i=2 L-3$, the equations of (A.1) simplifies into

$$
\left\{\begin{array}{l}
\beta_{13} E_{3}-\beta_{31} E_{1}=0 \\
\beta_{2 L-3,2 L-1} E_{2 L-1}-\beta_{2 L-1,2 L-3} E_{2 L-3}=0
\end{array}\right.
$$


As symmetric movement rates for boats were assumed, $\beta_{3,1}=\beta_{1,3}$ and, therefore, $E_{1}$ and $E_{3}$ must be equal at equilibrium and as a consequence, taking into account (13) and (14), we obtain for all $i$

$$
\left\{\begin{array}{l}
n_{i}^{*}=v_{i}^{*} n=\frac{k_{i}}{K} n=s_{i} K \\
E_{i}^{*}=\mu_{i}^{*} E=\frac{1}{L} E \quad \text { if area } i \text { is not in a reserve } \\
E_{i}^{*}=0 \quad \text { if area } i \text { is in a reserve }
\end{array}\right.
$$

(B) Derivation of the aggregated model

We substitute the fast equilibria $(A .4)$ into the complete model $(15,16,17,18)$, and add the $(2 L)$ fish stock and the $L$ fishing effort equations. After some algebra, one obtains the following system of two equations governing the total fish stock and fishing effort variables at the slow time scale, which we call the aggregated model:

$$
\left\{\begin{array}{l}
\frac{d n}{d t}=r n\left(1-\frac{n}{K}\right)-Q n E \\
\frac{d E}{d t}=(-c+p Q n) E
\end{array}\right.
$$

where $Q$ is as defined in the text.

\section{REFERENCES}

[1] M. T. Agardy, Advances in marine conservation: the role of marine protected areas. Trends in Ecology and Evolution, 9 (1994) 267-270.

[2] R. Hannesson, Marine reserves: what would they accomplish? Marine Resource Economics 13, (1998) 159-170.

[3] A. Auger and J. C. Poggiale, Aggregation and emergence in systems of ordinary differential equations. Math. Comput. Model. 27 No.4, (1998), 1-21.

[4] P. Auger, C. Lett, A. Moussaoui and S. Pioch, Optimal number of sites in artificial pelagic multisite fisheries. Can. J. Fish. Aquat. Sci. 67, (2010) 296-303.

[5] P. Auger and Bravo de la Parra, R, Methods of aggregation of variables in population dynamics. C. R. Acad. Sci. 323 (2000) 665-674.

[6] P. Auger and J. C. Poggiale. Emergence of population growth models: fast migration and slow growth. J. Theor. Biol. 182 (2), (1996) 99-108.

[7] P. Auger and J. C. Poggiale, Aggregation and emergence in systems of ordinary differential equations. Math. Comput. Model. 27 (4) 1998: 1-21.

[8] P. Auger, and R. Roussarie, Complex ecological models with simple dynamics: from individuals to population. Acta Biotheor. 42 (2-3), (1994) 111-136.

[9] P; Auger, R. Bravo de la Parra, J.C. Poggiale, E. Sanchez and T. Nguyen-Huu, Aggregation of variables and applications to population dynamics. In Structured population models in biology and epidemiology. Lecture notes in mathematics. Vol. 1936. Edited by P. Magal and S. Ruan. Mathematical Biosciences Subseries, Springer, Berlin. (2008) 209-263.

[10] M. Bensenane, A. Moussaoui and P. Auger, On the optimal size of marine reserves. Acta Biotheor. 61, (2013), 109-118.

[11] O. Chateau and L. Wantiez, Movement patterns of four coral reef fish species in a fragmented habitat in New Caledonia: implications for the design of marine protected area networks. ICES Journal of Marine Science. 66, (2009), 50-55.

[12] C. M. Clark, Mathematical bioeconomics: the optimal management of renewable resources. 2nd ed. Wiley InterScience, New York. (1990)

[13] J. M. Diamond, The island dilemma: lessons of modern biogeographic studies for the design of nature reserves. Biol. Conserv. 7, (1975) 129-146.

[14] J. M. Diamond, Island biogeography and conservation: strategy and limitations. Science. 193, (1976), $1027-1029$.

[15] L. Edelstein-Keshet, Mathematical Models in Biology. Random House, New York. (1998).

[16] M. E. Gilpin and J. M. Diamond, Subdivision of nature reserves and the maintenance of species diversity. Nature, 285, (1980), $567-568$.

[17] R. Groeneveld, Economic considerations in the optimal size and number of reserve sites. Ecological Economics 52, (2005), 219228.

[18] A. J. Higgs, M. B. Usher, Should nature reserves be big or small? Nature, 285, (1980) 568-569.

[19] D. S. Holland, and R. J. Brazee, Marine reserves for fishery management. Marine Resource Economics 11 (1996) $157-171$. 
[20] S. E. Kingsland, Creating a science of nature reserve design: Perspectives from history. Env Model Asses. 7, (2002), 61-69.

[21] J. Michalski, J.C. Poggiale, R. Arditi, and P. Auger, Macroscopic dynamic effects of migrations in patchy predator-prey systems. J. Theor. Biol. 185, (1997) 459-474.

[22] A. Moussaoui, P. Auger, C. Lett, Optimal number of sites in multi-site fisheries with fish stock dependent migrations. Mathematical Biosciences and Engineering, 8 (3), (2011), 769-783.

[23] A. Moussaoui, M. Bensenane, P. Auger, A. Bah, On the optimal size and number of reserves in a multi-site fishery model. Journal of Biological Systems, 22 (4) (2014), 1-17.

[24] J. C. Poggiale, Applications des Varietes Invariantes a la Modélisation de l'Hétérogenéité en Dynamique des Populations. Ph.D. Thesis, Bourgogne University, Dijon. In French. (1994).

[25] M. B. Schaefer, Some considerations of population dynamics and economics in relation to the management of the commercial marine fisheries. J. Fish. Res. Board Canada, 14, (1957), 669-681.

[26] V. L. Smith, On models of commercial fishing. J. Political Economy, 77 (2), (1969) 181-198.

[27] M. E. Soule and D. Simberloff. What do genetics and ecology tell us about the design of nature reserves? Biol. Conserv, 35 (1986), 19-40.

[28] B. A. Wilcox and D. D. Murthy, Conservation strategy: the effects of fragmentation on extinction. Am. Nat. 125, (1985) 879-887. 